\title{
The generalized cosmic equation of state: a revised study with cosmological standard rulers
}

\author{
Yubo $\mathrm{Ma}^{1}$, Jia Zhang ${ }^{2}$, Shuo $\mathrm{Cao}^{3, \text { a }}$, Xiaogang Zheng ${ }^{3}$, Tengpeng $\mathrm{Xu}^{3}$, Jingzhao $\mathrm{Qi}^{3}$ \\ ${ }^{1}$ School of Physics, Shanxi Datong University, Datong 037009, China \\ 2 Department of Physics, School of Mathematics and physics, Weinan Normal University, Weinan 714099, Shanxi, China \\ ${ }^{3}$ Department of Astronomy, Beijing Normal University, Beijing 100875, China
}

Received: 20 August 2017 / Accepted: 11 December 2017 / Published online: 20 December 2017

(C) The Author(s) 2017. This article is an open access publication

\begin{abstract}
In this paper, the generalized equation of state $(\mathrm{GEoS})$ for dark energy $\left(w_{\beta}=w_{0}-w_{\beta}\left[(1+z)^{-\beta}-1\right] / \beta\right)$ is investigated with the combined standard ruler data from the observations of intermediate-luminosity radio quasars, galaxy clusters, BAO and CMB. The constraint results show that the best-fit EoS parameters are $w_{0}=-0.94_{-0.41}^{+0.57}$, $w_{\beta}=-0.17_{-4.81}^{+2.45}$ and $\beta=-1.42$ (with a lower limit of $\beta>-2.70$ at $68.3 \%$ C.L.), which implies that at early times the dark energy vanishes. In the framework of nine truncated GEoS models with different $\beta$ parameters, our findings present very clear evidence disfavoring the case that dark energy always dominates over the other material components in the early universe. Moreover, stringent constraints can be obtained in combination with the latest measurements of Hubble parameter at different redshifts: $w_{0}=-1.01_{-0.31}^{+0.56}, w_{\beta}=0.01_{-4.52}^{+2.33}$ and $\beta=-0.42$ (with a lower limit of $\beta>-2.40$ at $68.3 \%$ C.L.). Finally, the results obtained from the transition redshift $\left(z_{t}\right)$ and $O m(z)$ diagnostic indicate that: (1) The above constraints on the GEoS model agree very well with the transition redshift interval $0.49 \leq z_{t} \leq 0.88$ within $1 \sigma$ error region. (2) At the current observational level, the GEoS model is practically indistinguishable from $\Lambda \mathrm{CDM}$, although a small deviation from $\Lambda \mathrm{CDM}$ cosmology is present in the combined standard ruler data.
\end{abstract}

\section{Introduction}

From many astrophysical and cosmological observations such as Type Ia Supernovae (SN Ia) [1-4] and cosmic microwave background radiation (CMB) [5-8], there is

\footnotetext{
a e-mail: caoshuo@bnu.edu.cn
}

strong evidence for dark energy (DE) with negative pressure, which dominates the total energy density and causes an accelerating expansion of our universe at late times. The most obvious theoretical candidate of dark energy is the cosmological constant $\Lambda$, which can fit the observations well, but which is plagued with the fine-tuning problem and the coincidence problem [9]. Numerous other dynamical DE models have also been proposed in the literature, such as the quintessence $[10,11]$, phantom $[12,13]$, quintom [14-16], Chaplygin gas model [17-19] and holographic models [20,21] etc.

Because dark energy possesses a negative pressure and therefore leads to a distinct equation of state $(\operatorname{EoS}): w=p / \rho$, which is quite different from the ordinary matter (cold dark matter plus baryons), much previous work has been done in terms of the modification and parameterizations of the EoS which incorporates the recent prediction from observational cosmology. The most often investigated EoS parameterizations are $w(z)=w_{0}+w_{P 1} z$ [22-24]; $w(z)=$ $w_{0}+w_{P 2} \ln (1+z)[25]$, and $w(z)=w_{0}+w_{P 3} z /(1+z)$ $[26,27]$, where $w_{0}$ is the current value of the EoS parameter, and $w_{\mathrm{P}}(\mathrm{P}=\mathrm{P} 1, \mathrm{P} 2, \mathrm{P} 3)$ are free parameters quantifying the time-dependence of the dark energy EoS, which must be constrained by various observational data. Note that the EoS associated with the $\Lambda \mathrm{CDM}$ model can be always recovered by taking $w_{\mathrm{P}}=0$ and $w_{0}=-1$ (see Ref. [28-32] for other parameterizations). Recently, Barboza et al. [33] proposed a generalized cosmic equation of state (GEoS) expressed as

$w(z)=w_{0}-w_{\beta} \frac{(1+z)^{-\beta}-1}{\beta}$.

From the above expressions, it is straightforward to show that the above EoS parameterizations are fully recovered when $\beta \rightarrow-1.0, \beta \rightarrow 0$ and $\beta \rightarrow+1.0$, respectively. Therefore, the introduction of the new parameter $\beta$ is equivalent to 
putting the EoS parameterizations (P1)-(P3) in a more general framework allowing more categories of cosmological solutions [33].

From the energy conservation law, it is easy to show that the ratio $f_{\beta}=\rho_{\beta} / \rho_{\beta}^{0}$ evolves as [33]

$f_{\beta}=(1+z)^{3\left(1+w_{0}+w_{\beta} / \beta\right)} \exp \left[\frac{3 w_{\beta}}{\beta}\left(\frac{(1+z)^{-\beta}-1}{\beta}\right)\right]$.

Note that some cases of interest relating the parameters $w_{0}$, $w_{\beta}$ and $\beta$ may also be obtained:

1. $\beta>0\left(w_{\beta}<0\right.$ or $\left.w_{\beta}>0\right)$ : At early times the dark energy is a subdominant component if $w_{0}+w_{\beta} / \beta \leq 0$.

2. $\beta<0$ and $w_{\beta}>0$ : At early times the dark energy always dominates over the other material components.

3. $\beta<0$ and $w_{\beta}<0$ : At early times the dark energy density vanishes.

Currently, much work has been done on this generalized EoS model with the attempt to constrain it by using various observational data, such as SN Ia and the Hubble parameter data [33]. On the other hand, recently, the angular size of the compact structure in radio quasars versus redshift data from the very-long-baseline interferometry (VLBI) observations has become an effective probe in cosmology. Reliable standard rulers at cosmological scales are crucial for measuring cosmic distances at different redshifts. In the past, there have been controversies as regards whether the compact radio sources could act as standard rulers [34-40]. The difficulty lies in the fact that the linear sizes $l_{m}$ of compact radio sources might not be constant, i.e., its value is dependent on both redshifts and some intrinsic properties of the source (luminosity, for example). Based on a $2.29 \mathrm{GHz}$ VLBI all-sky survey of 613 milliarcsecond ultra-compact radio sources, Cao et al. [41] found that only intermediate-luminosity quasars show negligible dependence on both redshifts $z$ and intrinsic luminosity $L$, which makes it possible for them to perform as standard rulers with a fixed comoving length. More recently, based on a cosmological-model-independent method to calibrate the linear sizes $l_{m}$ of compact radio quasars, Cao et al. [42] investigated the cosmological application of this data set and obtained stringent constraints on both the matter density, $\Omega_{m}$, and the Hubble constant, $H_{0}$, which agree very well with those derived from the recent Planck measurements. The advantage of this data set, compared with other standard rulers including baryon acoustic oscillations (BAO) [43-46], galaxy clusters [47] and strong lensing systems [48-53], is that quasars are observed at much higher redshifts $(z \sim 3.0)$. However, in order to consider the redshift coverage of observations and break the degeneracy of the model parameters, we also add, in our discussion, the angular diameter distance measurements from galaxy clusters, BAO and CMB, which have been extensively used to probe the nature of cosmic acceleration in much previous work [54-57].

This paper is organized as follows: In Sect. 2, we introduce the latest data sets used in our analysis. In Sect. 3, we show the constraint results from standard ruler data and furthermore apply the transition redshift interval data and model diagnostics in Sect. 4. Finally, we summarize the main conclusions in Sect. 5.

\section{Observational data and analysis}

\subsection{Quasars data}

The BAO peak location is commonly recognized as a fixed comoving ruler of about $100 \mathrm{Mpc}$ [43-46]. In a similar spirit, as extensively discussed in the literature, compact radio sources (quasars, in particular) constitute another possible class of standard rulers with the comoving length of about 10 pc. Following the analysis of Gurvits [35], luminosity and redshift dependence of the linear sizes of quasars can be parametrized as

$l_{m}=l L^{m}(1+z)^{n}$

where $m$ and $n$ are two parameters quantifying the "angular size-redshift" and "angular size-luminosity" relations, respectively. The parameter $l$ is the linear size scaling factor representing the apparent distribution of radio brightness within the core. The data used in this paper were derived from an old 2.29 GHz VLBI survey presented by Preston et al. [58], which contains 613 milliarcsecond ultra-compact radio sources covering the redshift range $0.0035<z<$ 3.787. More recently, Cao et al. [41] presented a method to identify a sub-sample which can serve as a certain class of individual standard rulers in the universe. According to the optical counterparts and luminosities, the full sample could be divided into three sub-samples: low-luminosity quasars $\left(L<10^{27} \mathrm{~W} / \mathrm{Hz}\right)$, intermediate-luminosity quasars $\left(10^{27} \mathrm{~W} / \mathrm{Hz}<L<10^{28} \mathrm{~W} / \mathrm{Hz}\right)$ and high-luminosity quasars $\left(L>10^{28} \mathrm{~W} / \mathrm{Hz}\right)$. The final results showed that only intermediate-luminosity quasars show negligible dependence $\left(|n| \simeq 10^{-3},|m| \simeq 10^{-4}\right)$, and thus they could be a population of rulers once the characteristic length $l_{m}$ is determined. In our analysis, we will use the observations of 120 intermediate-luminosity quasars covering the redshift range $0.46<z<2.80$, while the linear size of this standard ruler is calibrated to $l_{m}=11.03 \pm 0.25 \mathrm{pc}$ through a new cosmologyindependent technique [42].

The observable quantity in this data set is the angular size of the compact structure in intermediate-luminosity radio 
Table 1 The currently available angular diameter distance data from BAO and their references

\begin{tabular}{lllllll}
\hline$z$ & 0.35 & 0.44 & 0.57 & 0.60 & 0.73 & 2.34 \\
\hline$D_{A}(\mathrm{Mpc})$ & 1050 & 1205 & 1380 & 1380 & 1534 & 1662 \\
$1 \sigma(\mathrm{Mpc})$ & \pm 38 & \pm 114 & \pm 23 & \pm 95 & \pm 107 & \pm 96 \\
Ref. & Blake et al. [43] & Blake et al. [43] & Blake et al. [43] & Xu et al. [44] & Samushia et al. [45] & Delubac et al. [46] \\
\hline
\end{tabular}

quasars, whose theoretical (i.e. determined by the cosmological model) counterpart is

$\theta_{\mathrm{th}}(z)=\frac{l_{m}}{D_{A}(z)}$

where $D_{A}$ is the angular diameter distance at redshift $z$ and the GEoS parameters $\mathbf{p}$ directly enter the angular diameter distance through

$D_{A}(z ; \mathbf{p})=\frac{c}{(1+z) H_{0}} \int_{0}^{z} \frac{\mathrm{d} z^{\prime}}{E\left(z^{\prime} ; \mathbf{p}\right)}$

where $H_{0}$ is the Hubble constant and $E\left(z^{\prime} ; \mathbf{p}\right)$ is the dimensionless Hubble parameter. For the quasar sample, the corresponding $\chi^{2}$ is defined as

$\chi_{\mathrm{QSO}}^{2}(z ; \mathbf{p})=\sum_{i=1}^{120} \frac{\left[\theta_{\mathrm{th}}\left(z_{i} ; \mathbf{p}\right)-\theta_{\mathrm{obs}}\left(z_{i}\right)\right]^{2}}{\sigma_{\theta}\left(z_{i}\right)^{2}}$

where $\theta_{\mathrm{obs}}\left(z_{i}\right)$ is the observed value of the angular size and $\sigma_{\theta}\left(z_{i}\right)$ is the corresponding uncertainty for the $i$ th data point in the sample. In order to properly account for the intrinsic spread in linear sizes and systematics we have added in quadrature $10 \%$ uncertainties to the $\sigma_{\theta}\left(z_{i}\right)$ [42].

\subsection{Other standard ruler data}

In order to diminish the degeneracy between model parameters we also used the accurate measurements of angular diameter distances from galaxy clusters, BAO and CMB. More importantly, the combination of high-redshift quasars and other observational probes at lower redshifts could result in a fair coverage of redshifts $[42,59,60]$.

The measurements of BAO in the large-scale structure power spectrum have also been widely used for cosmological applications. In this work we consider the six measurements of $D_{A}$ in several previous works [43-46]. These data and their references are listed in Table 1. The detailed information as regards these data can be found in these references. We can obtain the angular diameter distances by using the SunyaevZeldovich effect together with X-ray emission of 25 galaxy clusters $(0.14<z<089)$ [47], where an isothermal elliptical $\beta$ model was used to describe their mass distribution. For the above two astrophysical probes, cosmological parameters can be directly estimated by minimizing the corresponding $\chi^{2}$ as

$\chi_{\mathrm{BAO} / \text { Cluster }}^{2}=\sum^{i} \frac{\left[D_{A}^{\mathrm{th}}\left(z_{i} ; \mathbf{p}\right)-D_{A}^{\mathrm{obs}}\left(z_{i}\right)\right]^{2}}{\sigma_{D_{A}, i}^{2}}$.

Here $D_{A}^{\text {th }}\left(z_{i} ; \mathbf{p}\right)$ is the theoretical angular diameter distance at redshift $z_{i}$, which is defined in Eq. (5). $D_{A}^{\mathrm{obs}}\left(z_{i}\right)$ is the observed angular diameter distance of the $i$ th $\mathrm{BAO}$ or galaxy cluster with total uncertainty $\sigma_{D_{A}, i}^{2}$.

For the CMB data, the measurements of several quantities derived from Planck [61] will be used, which include the acoustic scale $\left(l_{A}\right)$, the shift parameter $(R)$, and the baryonic matter fraction at the redshift of recombination $\left(\Omega_{b 0} h^{2}\right)$. Firstly, the acoustic scale is expressed as

$l_{A} \equiv\left(1+z_{*}\right) \frac{\pi D_{A}\left(z_{*}\right)}{r_{s}\left(z_{*}\right)}$

where the redshift of the photon-decoupling period can be calculated as [62]

$z_{*}=1048\left[1+0.00124\left(\Omega_{b 0} h^{2}\right)^{-0.738}\right]\left[1+g_{1}\left(\Omega_{m 0} h^{2}\right)^{g_{2}}\right]$,

$g_{1}=\frac{0.0783\left(\Omega_{b 0} h^{2}\right)^{-0.238}}{1+39.5\left(\Omega_{b 0} h^{2}\right)^{0.763}}, g_{2}=\frac{0.560}{1+21.1\left(\Omega_{b 0} h^{2}\right)^{1.81}}$.

The comoving sound horizon can be parameterized as

$$
\begin{aligned}
r_{s}\left(z_{*}\right) & =\int_{0}^{t} \frac{c_{s} \mathrm{~d} t^{\prime}}{a}=\frac{c}{H_{0}} \int_{z_{*}}^{\infty} \frac{c_{s} \mathrm{~d} z}{E(z)} \\
& =\frac{c}{H_{0}} \int_{0}^{a_{*}} \frac{\mathrm{d} a}{a^{2} E(a) \sqrt{3\left(1+\overline{R_{b}} a\right)}},
\end{aligned}
$$

with $\overline{R_{b}}=31500\left(T_{\mathrm{CMB}} / 2.7 K\right)^{-4} \Omega_{b 0} h^{2}$ and $T_{\mathrm{CMB}}=$ $2.7255 \mathrm{~K}$. Note that the current radiation component is related to the matter density as $\Omega_{r 0}=\Omega_{m 0} /\left(1+z_{e q}\right)$, where $z_{\text {eq }}=2.5 \times 10^{4} \Omega_{m 0} h^{2}\left(T_{\mathrm{CMB}} / 2.7 K\right)^{-4}$. Secondly, the other commonly used CMB shift parameter reads [63]

$R\left(z_{*}\right) \equiv \frac{\left(1+z_{*}\right) D_{A}\left(z_{*}\right) \sqrt{\Omega_{m 0} H_{0}^{2}}}{c}$. 

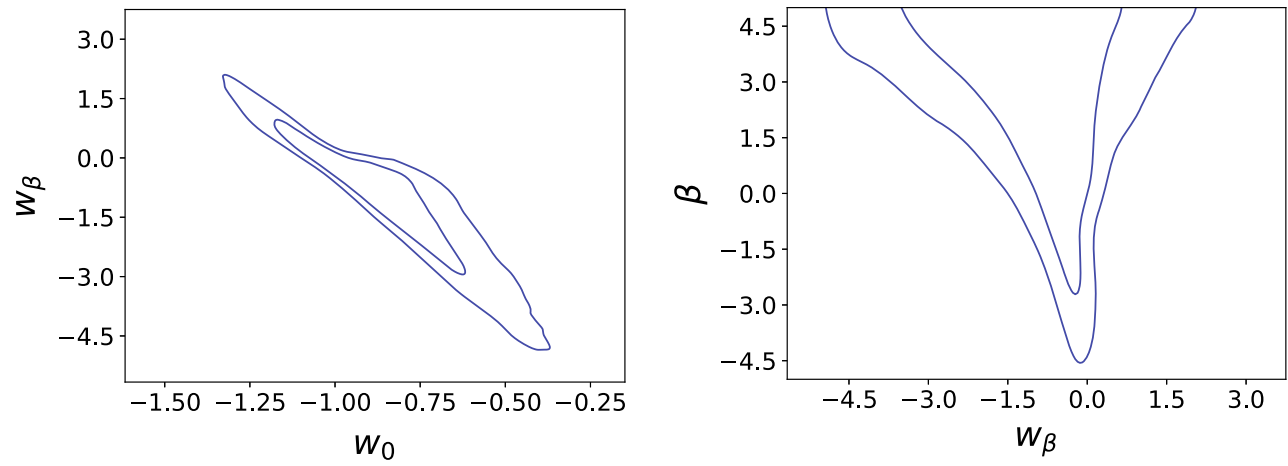

Fig. 1 The 68.3 and $95.4 \%$ confidence level contours for $w_{0}$ versus $w_{\beta}$ and $w_{\beta}$ versus $\beta$ for the standard ruler data. The best fit happens at $w_{0}=-0.94, w_{\beta}=0.17$ and $\beta=-1.42$

Model parameters can be estimated from the CMB data using the likelihood based on the $\chi^{2}$ function defined as

$\chi_{\mathrm{CMB}}^{2}=\Delta P_{\mathrm{CMB}}^{T} C_{\mathrm{CMB}}^{-1} \Delta P_{\mathrm{CMB}}$

where $\Delta P_{\mathrm{CMB}}$ is the difference between the theoretical distance priors and the observational counterparts. The corresponding inverse covariance matrix $C_{\mathrm{CMB}}^{-1}$ can be found in Table 4 provided by Ade et al. [61].

We have incorporated the three standard ruler probes into the analysis of quasar data and the total likelihood is taken to be the products of the separate likelihoods of all cosmological probes:

$\chi_{\mathrm{tot}}^{2}=\chi_{\mathrm{QSO}}^{2}+\chi_{\mathrm{Cluster}}^{2}+\chi_{\mathrm{BAO}}^{2}+\chi_{\mathrm{CMB}}^{2}$.

The best-fit model parameters are determined by minimizing the $\chi_{\text {tot }}^{2}$ and confidence limits on the model parameters are obtained by using the usual $\Delta \chi^{2}\left(\equiv \chi^{2}-\chi_{\min }^{2}\right)$ statistics, where $\chi_{\min }^{2}$ is the global minimum $\chi^{2}$ for the best-fit parameters. Our calculating method is based on a Markov Chain Monto Carlo (MCMC) method; the analysis is performed on the publicly available CosmoMC package [64].

\section{Constraints on the generalized EoS model}

For the generalized $w_{\beta}(z)$ model, the Friedmann equation for a spatially flat universe which contains only dust matter (cold dark matter plus baryons) and dark energy, can be expressed as

$$
\begin{aligned}
E(z ; \mathbf{p}) & =H / H_{0} \\
& =\sqrt{\Omega_{m}(1+z)^{3}+\left(1-\Omega_{m}\right) f_{\beta}},
\end{aligned}
$$

Therefore, there are five independent model parameters $\mathbf{p} \equiv$ $\left(\Omega_{m}, w_{0}, w_{\beta}, \beta, H_{0}\right)$ in this model.

For this combined standard ruler data, the best fit values for the GEoS parameters are $\Omega_{m}=0.304_{-0.013}^{+0.016}, w_{0}=$
$-0.94_{-0.41}^{+0.57}, w_{\beta}=-0.17_{-4.81}^{+2.45}, \beta=-1.42$, and $H_{0}=$ $68.43_{-0.71}^{+0.75} \mathrm{~km} / \mathrm{s} / \mathrm{Mpc}$ at $68.3 \%$ confidence level. Figure 1 shows the contour plot (68.3 and $95.4 \%$ confidence levels) in the $w_{0}-w_{\beta}$ and $w_{\beta}-\beta$ planes for the $\chi_{\text {total }}^{2}$ given by Eq. (14), which indicates that at early times the dark energy vanishes. Concerning the value of $\beta$, this result marginally disagrees with the recent analysis of Barboza et al. [33], which incorporated the joint observations including the Constitution set of $397 \mathrm{SN}$ Ia, the CMB shift parameter $R$, the BAO parameter $A$, and nine determinations of the Hubble parameter from the differential ages of red-envelope galaxies [33]: $w_{0}=-0.98$, $w_{\beta}=0.1, \beta=-3.04(\mathrm{SN} \mathrm{Ia}+\mathrm{BAO}+H(z))$ and $w_{0}=-1.0$, $w_{\beta}=0.28, \beta=0.1$ (SN Ia+BAO+CMB) [33]. Apparently, these combined data only give a relatively weak constraint on the model parameters, a result consistent with those obtained with other observational data sets [33]. However, we still obtain a lower limit for the $\beta$ parameter, $\beta>-2.70$ at $68.3 \%$ C.L. Moreover, when $\beta<-1$, the EoS parameter $w_{\beta}$ might be reduced into a narrow strip. Therefore, $\beta$ can play an significant part amid the parameter estimations. In order to gain an insight into this conclusion, we assign different values to this parameter with results shown in Fig. 2, which exhibits variations of $w_{\beta}$ with $w_{0}$ in the truncated planes $\left(w_{0}-w_{\beta}\right)$. In the dark energy model with generalized equation of state, the Chevalier-Polarski-Linder (CPL) parameterizations could be fully recovered when $\beta=+1.0$. One can deduce that this model is only one specific projection in different parameter subspaces of the general EoS model. Considering the physical meaning of $\beta$ quantifying the dynamical properties of dark energy, we assign different values $\beta= \pm 2.0, \pm 1.5, \pm 1.0, \pm 0.5,0$ to this parameter and obtain nine time-dependent EoS parameterizations. Evidently, the greatest improvement in the parameter constraints is apparent around $\beta=-1$, because when $\beta<-1$ (clearly an upper limit), contours at $1 \sigma$ as well as $2 \sigma$ error regions are both greatly reduced. For the eight EoS scenarios, and $H_{0}$, the best-fit values of the EoS parameters $\left(w_{0}, w_{\beta}\right)$ with $1 \sigma$ uncertainties are presented in Fig. 2. 

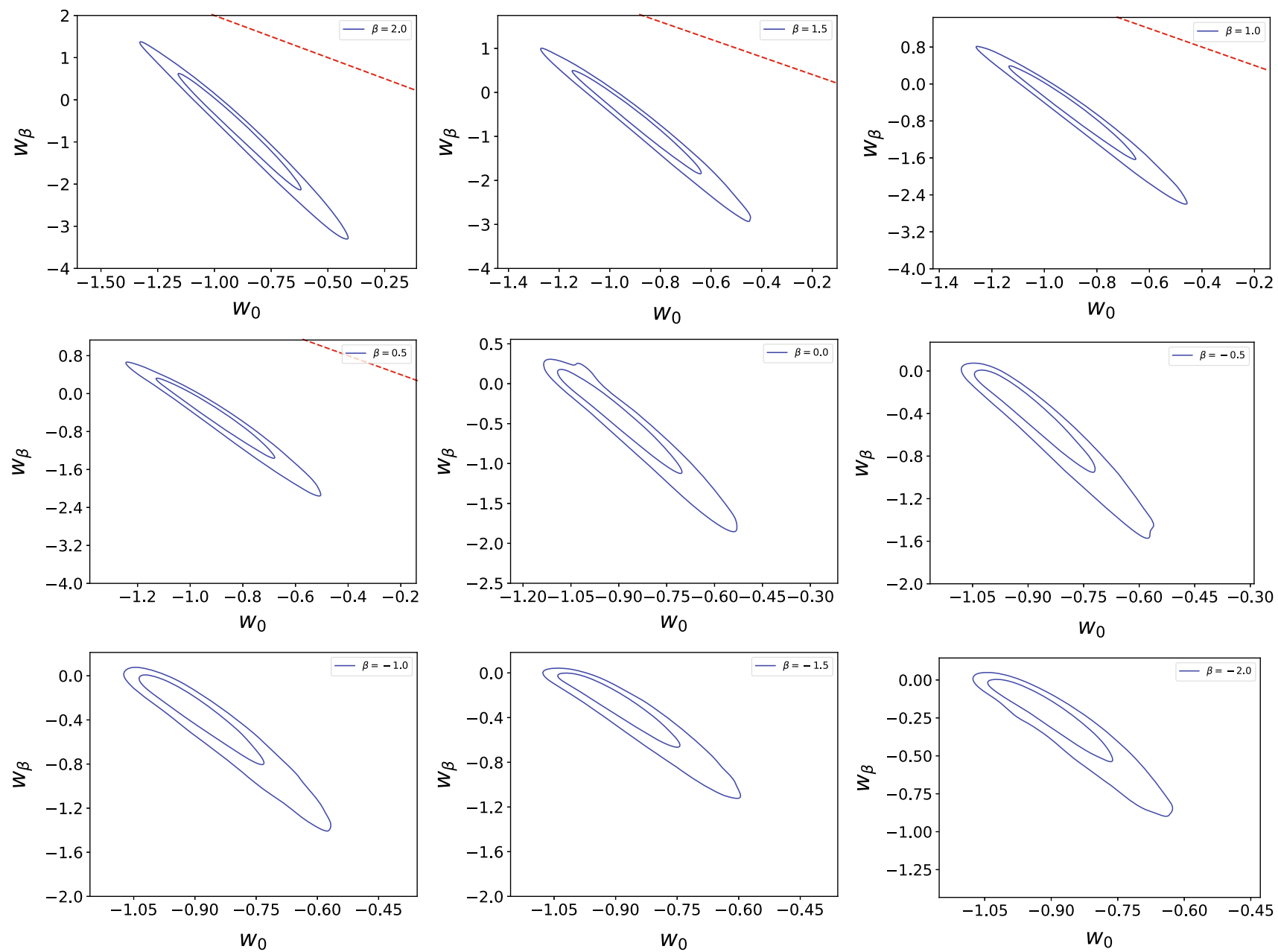

Fig. 2 The truncated planes $w_{0}-w_{\beta}$ with different truncated $\beta$ parameters. The special case with $w_{0}+w_{\beta} / \beta=0$ denoted with red dashed lines is also added for comparison

When the $\beta$ parameter is fixed at $\beta=+2.0$ and the cosmic equation of state takes the form $w(z)=w_{0}-w_{2}[(1+$ $\left.z)^{-2}-1\right] / 2$, the best-fit values for the EoS parameters are $w_{0}=-0.88_{-0.37}^{+0.40}, w_{2}=-0.70_{-2.21}^{+1.69}$ at $68.3 \%$ confidence level. Working on the commonly used CPL parametrization with $\beta=+1.0$, we obtain $w_{0}=-0.92_{-0.31}^{+0.38}$ and $w_{1}=-0.45_{-1.76}^{+1.14}$, from which one can see fairly good agreement between our results obtained by using the standard ruler data and the concordance $\Lambda$ CDM model. Performing a similar analysis on the cosmic equation of state with $\beta=+1.5$ and $\beta=-0.5$, we derive the best-fit parameters as $w_{0}=-0.91_{-0.32}^{+0.39}, w_{1.5}=-0.52_{-1.97}^{+1.35} ; w_{0}=$ $-0.93_{-0.27}^{+0.36}, w_{0.5}=-0.35_{-1.49}^{+0.88}$; and $w_{0}=-0.94_{-0.18}^{+0.35}$, $w_{0.0}=-0.27_{-1.31}^{+0.46}$. Concerning the physical meaning of different combination of $w_{0}, w_{a}$ and $\beta$, in order to illustrate the role of dark energy played at early times of the universe, we also show the special case with $w_{0}+w_{\beta} / \beta=0$ in Fig. 2, which strongly indicates that at early times the dark energy is a subdominant component at the $95.4 \%$ confidence level. Next, we examine the cosmic equation of state which allows the $\beta$ parameter to be smaller than zero. When $\beta<0$, contours of the EoS parameters at $1 \sigma$ as well as $2 \sigma$ error regions are both greatly reduced: $w_{0}=-0.95_{-0.12}^{+0.33}, w_{-0.5}=$ $-0.20_{-1.13}^{+0.20} ; w_{0}=-0.93_{-0.14}^{+0.30}, w_{-1}=-0.21_{-0.92}^{+0.21} ; w_{0}=$ $-0.95_{-0.12}^{+0.29}, w_{-1.5}=-0.14_{-0.80}^{+0.14}$; and $w_{0}=-0.97_{-0.10}^{+0.29}$, $w_{-2}=-0.09_{-0.67}^{+0.09}$. Focusing on the physical meaning of different combination of $w_{0}, w_{\beta}$ and $\beta$, our results with negative $\beta$ parameters tend to support the indication that the dark energy density vanishes at early times (at $68.3 \%$ confidence level).

In performing the statistical analysis from the standard ruler data set, we find that the standard rulers will shift the matter density parameter to a higher value $\left(\Omega_{m} \sim 0.304\right)$ and a lower value for the Hubble constant $\left(H_{0} \sim 68.45\right.$ $\mathrm{km} / \mathrm{s} / \mathrm{Mpc}$ ), which is well consistent with that obtained from the independent measurements including the recent Planck CMB [61] and radio quasar data [42], but essentially different from the corresponding fits derived from standard candles 

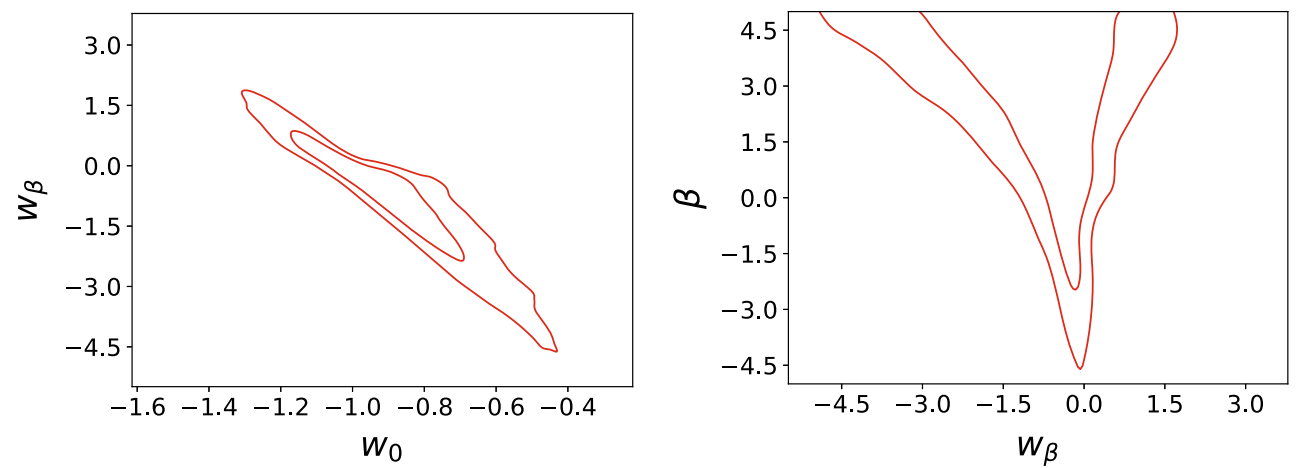

Fig. 3 The 68.3 and $95.4 \%$ confidence level contours for $w_{0}$ versus $w_{\beta}$ and $w_{\beta}$ versus $\beta$ for the combined standard ruler and standard clock data. The best fit happens at $w_{0}=-1.01, w_{\beta}=0.01$ and $\beta=-0.42$

(SN Ia) $[65,66]$. Moreover, the parameter $\beta$ can play an significant part amid the parameter estimations, i.e., with the decrease of $|\beta|$, the other EoS parameter characterizing the evolution of $w$ evolving with redshift, $w_{\beta}$, will be more stringently constrained. Finally, it is implied that at early times the dark energy is a subdominant component (at the $95.4 \%$ confidence level) or vanishes (at the $68.3 \%$ confidence level), i.e., the special case that at early times the dark energy always dominates over the other material components is not preferred by the available observations.

From the observational point of view, the ages of passively evolving early-type galaxies will provide direct measurements of the Hubble parameter $H(z)$ at different redshifts, which constitute another type of standard probe (i.e., standard clocks) in cosmology [67]. Moreover, compared with other types of observational data, it is more rewarding to investigate the observational $H(z)$ data directly, because it can take the fine structure of $H(z)$ into consideration and then use the important information compiled in it. It should be noted that $H(z)$ measurements are always obtained from two different techniques: galaxy differential age, also known as cosmic chronometer (CC) and radial BAO size methods. The latest $36 H(z)$ data set including $30 \mathrm{CC} H(z)$ data and six BAO $H(z)$ data is compiled in Table 1 of Zheng et al. [68]. In this work, considering the correlation between $H(z)$ and BAO, we only use 30 Hubble parameter measurements obtained from cosmic chronometers, i.e. massive, early-type galaxies evolving passively on a timescale longer than their age difference. The $\chi^{2}$ statistics for these $H(z)$ data covering the redshift range of $0.07<z<1.965$ is defined as

$\chi_{H}^{2}=\sum_{i=1}^{30} \frac{\left(H\left(\mathbf{p} ; z_{i}\right)-H_{i}\right)^{2}}{\sigma_{H, i}^{2}}$,

where $\sigma_{H, i}$ is the $1 \sigma$ uncertainty in the $H(z)$ data.

Fitting the GEoS model to the combined standard ruler and standard clock data, we obtain $\Omega_{m}=0.310_{-0.019}^{+0.008}$, $w_{0}=-1.01_{-0.31}^{+0.56}, w_{\beta}=0.01_{-4.52}^{+2.33}, \beta=-0.42$ and $H_{0}=68.40_{-0.67}^{+0.81} \mathrm{~km} / \mathrm{s} / \mathrm{Mpc}$. Figure 3 shows the contour plot (68.3 and $95.4 \%$ confidence levels) in the $w_{0}-w_{\beta}$ and $w_{\beta}-\beta$ planes for this joint analysis. Apparently, the combination with all available observational data gives a more stringent constraint on the model parameters, i.e., a lower limit for the $\beta$ parameter, $\beta>-2.40$ is also determined at $68.3 \%$ C.L. More interestingly, the implication that the dark energy vanishes at early times is still supported at the current observational level. Therefore, it is reasonable to expect that a large amount of the observational $H(z)$ data will dramatically minimize systematic uncertainties by design, and thus shed much more light into the dark universe in the future [69].

\section{Model diagnostics}

In order to better understand the nature of dark energy, it is important to apply some sensitive and robust diagnostics to illustrate the dynamic behavior of dark energy with generalized equation of state.

In this way, we first derive the deceleration parameter to study the influence of the parameter $\beta$ on the epoch of cosmic acceleration

$q(z)=\frac{1}{2} \frac{\Omega_{m}(1+z)^{3}+\left(1-\Omega_{m}\right)\left(f_{\beta}^{\prime}(1+z)-2 f_{\beta}\right)}{\Omega_{m}(1+z)^{3}+\left(1-\Omega_{m}\right) f_{\beta}}$,

where $f_{\beta}^{\prime}(1+z)=3\left(1+w_{0}+\frac{w_{\beta}}{\beta}\right) f_{\beta}-\frac{3 w_{\beta}(1+z)^{-\beta}}{\beta} f_{\beta}$. The transition redshift $z_{t}$, at which the universe switches from deceleration to acceleration, is the very redshift corresponding to $q(z)=0$. Figure 4 shows the deceleration parameter $q(z)$ as a function of redshift with the best-fitted value as well as the $1 \sigma$ uncertainties derived from the standard ruler data and combined standard probe data (standard ruler and standard clock $)^{1}$. The vertical lines stand for the interval

\footnotetext{
${ }^{1}$ Considering the fact that all of the current observations only place a lower limit on the $\beta$ parameter, we just take into account the $1 \sigma$ uncertainties of the other four model parameters $\left(\Omega_{m}, w_{0}, w_{\beta}, H_{0}\right)$.
} 


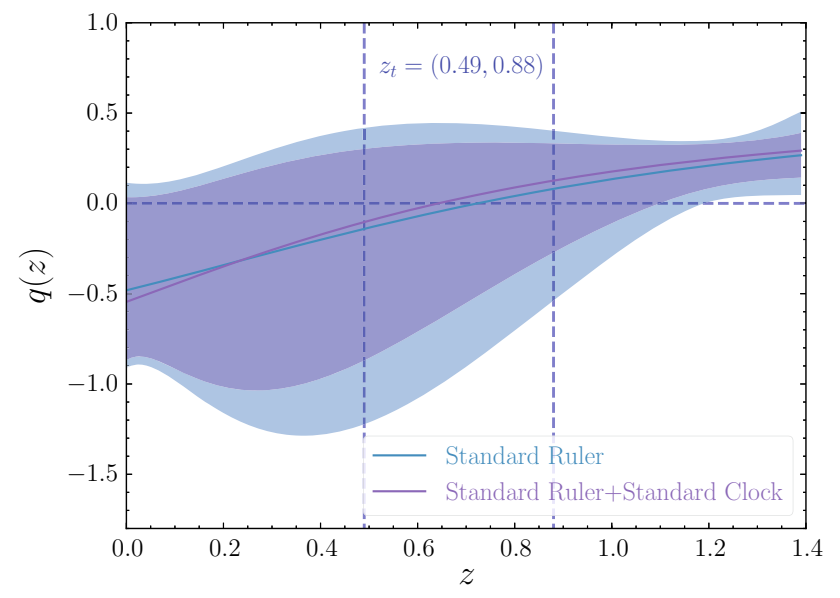

Fig. 4 Evolution of the deceleration parameter for the GEoS model from the standard ruler data (blue line), as well as the combined standard ruler and standard clock data (purple line). The $1 \sigma$ uncertainties are, respectively, denoted by blue and purple shades. The dashed vertical lines stand for the interval $0.49 \leq z_{t} \leq 0.88$, which corresponds to $\pm 1 \sigma$ of one of the values for $z_{t}$ estimated by Cunha [70]

$0.49 \leq z_{t} \leq 0.88$, which corresponds to $\pm 1 \sigma$ of the value for $z_{t}$ given by Cunha [70]. In particular, the cosmological results from dark energy with a generalized equation of state are in agreement with these transition redshift data within a $1 \sigma$ error region.

Moreover, it is well known that $\operatorname{Om}(z)$ provides a null test for the base $\Lambda \mathrm{CDM}$ cosmology and other evolving dark energy models [71,72], the expression of which is related to the Friedmann equation as

$O m(z)=\frac{E^{2}(z)-1}{(1+z)^{3}-1}$

It is apparent that in the base $\Lambda \mathrm{CDM}$ model neglecting the radiation at low redshifts, the value of $\operatorname{Om}(z)$ should be constant and exactly equal to the present mass density $\Omega_{m}$, while for other cosmological models, the $O m(z)$ diagnostic is expected to give different values. Therefore, $\operatorname{Om}(z)$ performs the most popular probe used to test the $\Lambda$ CDM model and to seek evidence of evolving cosmic equation of state $[68,73]$.

Applying the $O m(z)$ diagnostic to the GEoS model considered in our work, we can get the relation between the redshift and $\operatorname{Om}(z)$ for the GEoS model, which is specifically presented in Fig. 5. Another impressive feature of Fig. 5 is the slope of $\mathrm{Om}(z)$. If the value of $\mathrm{Om}(z)$ is a constant at any redshift, dark energy is exactly in the form of the cosmological constant, whereas the evolving $\operatorname{Om}(z)$ corresponds to other dynamical dark energy models. On the other hand, the slope of $\mathrm{Om}(z)$ could distinguish two different types of dark energy models, i.e., a positive slope indicates a phase of phantom $(w<-1)$ while a negative slope represents quintessence

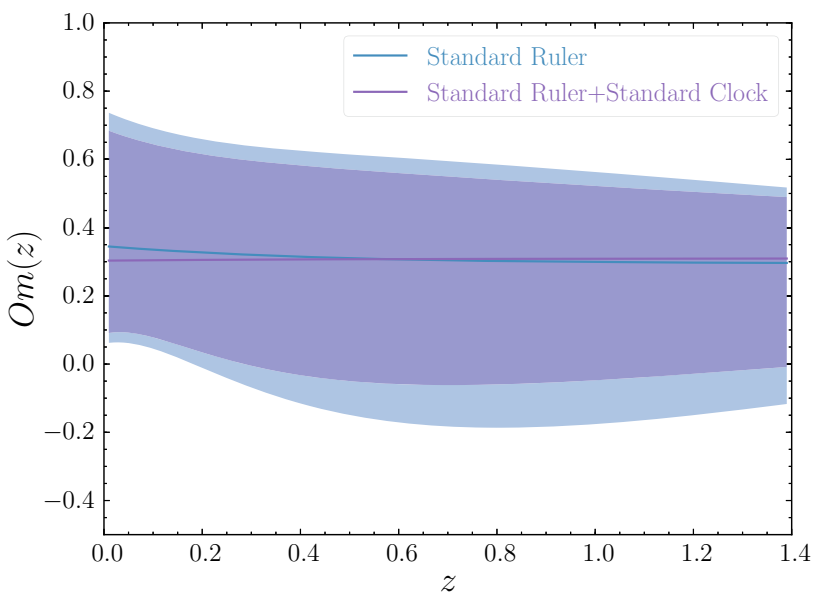

Fig. 5 The evolution of $O m(z)$ versus the redshift $z$ for the GEoS model from the standard ruler data (blue line), as well as the combined standard ruler and standard clock data (purple line). The $1 \sigma$ uncertainties are, respectively, denoted by blue and purple shades

$(w>-1)$ [72]. It is apparent that, in the framework of the GEoS models with the best-fit parameters from the standard ruler data, the slope of $\mathrm{Om}(z)$ is slightly smaller than zero, which suggests that the current observational data tend to support a quintessence cosmology. However, the combination of standard clock data implies that the GEoS model is practically indistinguishable from $\Lambda \mathrm{CDM}$. Such a tendency is more obvious when the $1 \sigma$ uncertainty of the model parameters is taken into consideration.

\section{Conclusions}

In this paper, we have examined, with the combined angular diameter distance data from the observations of milliarcsecond compact structure in intermediate-luminosity radio quasars, galaxy clusters, $\mathrm{BAO}$ and $\mathrm{CMB}$, to place constraints on the generalized EoS for dark energy. Furthermore, we have used sensitive and robust diagnostics to illustrate the dynamic behavior of dark energy generalized EoS. Here we summarize our main conclusions in more detail:

- Performing the statistical analysis from the standard ruler data set, we derive the best-fit EoS parameters as $w_{0}=-0.94_{-0.41}^{+0.57}, w_{\beta}=-0.17_{-4.81}^{+2.45}$ and $\beta=-1.42$, which implies that at early times the dark energy vanishes. Meanwhile, although these combined data fail to place very stringent constraints on the model parameters, $w_{0}, w_{\beta}$ and $\beta$, which is consistent with those obtained with other observational data constraints [33], for the $\beta$ parameter a lower limit of $\beta>-2.70$ is still determined at $68.3 \%$ C.L. in this work. Concerning the value of $\beta$, our results marginally disagree with the recent analysis 
of Barboza et al. [33], which incorporated the joint observations including the Constitution set of 397 SN Ia, the CMB shift parameter $R$, the BAO parameter $A$, and nine determinations of the Hubble parameter from the differential ages of red-envelope galaxies [33].

- Considering the physical meaning of $\beta$ quantifying the dynamical properties of dark energy, we assign different values of $\beta$ to this parameter and obtain nine time-dependent EoS parameterizations. For the truncated GEoS models, the standard rulers will shift the standard rulers will shift the matter density parameter to a higher value $\left(\Omega_{m} \sim 0.304\right)$ and a lower value for the Hubble constant $\left(H_{0} \sim 68.45 \mathrm{~km} / \mathrm{s} / \mathrm{Mpc}\right)$, which is well consistent with that obtained from the independent measurements including the recent Planck CMB [61] and radio quasar data [42], but which is essentially different from the corresponding fits derived from standard candles (SN Ia) $[65,66]$. Moreover, with the decrease of $|\beta|$, the other EoS parameters characterizing the evolution of $w$ evolving with redshift, $w_{\beta}$, will be more stringently constrained. More importantly, it is implied that at early times the dark energy is a subdominant component (at the $95.4 \%$ confidence level) or vanishes (at the $68.3 \%$ confidence level), i.e., the special case that at early times the dark energy always dominates over the other material components is not preferred by the available observations.

- Moreover, stringent constraints can be obtained in combination with other observational data including the measurements of Hubble parameter $H(z)$ at different redshifts. For the combined standard ruler and standard clock data, the best fit takes place at $w_{0}=-1.01_{-0.31}^{+0.56}$, $w_{\beta}=0.01_{-4.52}^{+2.33}$, and $\beta=-0.42$. Similarly, a lower limit for the $\beta$ parameter, $\beta>-2.40$ is also determined at $68.3 \%$ C.L. More interestingly, the implication that the dark energy vanishes at early times is still supported at the current observational level.

- In terms of the transition redshift $z_{t}$, at which the universe switches from deceleration to acceleration, we find that the results from the GEoS model are in consistence with the transition redshift data within a $1 \sigma$ error region. Applying the $O m(z)$ diagnostic to the GEoS model considered in our work, we find that, in the framework of the GEoS model with the best-fit parameters from the standard ruler data, the slope of $O m(z)$ is slightly smaller than zero, which suggests that the current observational data tend to support a quintessence cosmology. However, the combination of standard clock data implies that the GEoS model is practically indistinguishable from $\Lambda \mathrm{CDM}$, a tendency more obvious when the $1 \sigma$ uncertainties of model parameters are taken into consideration. Therefore, the above conclusions still need to be checked with future observational data such as highredshift radio quasars from the VLBI surveys [74,75], which will provide much better and competitive constraints on the dynamical properties of dark energy.

Acknowledgements This work was supported by National Key R\&D Program of China No. 2017YFA0402600, the Young Scientists Fund of the National Natural Science Foundation of China (Grant Nos. 11605107 and 11503001), in part by the National Natural Science Foundation of China (Grant No. 11475108), Supported by Program for the Innovative Talents of Higher Learning Institutions of Shanxi, the Natural Science Foundation of Shanxi Province, China (Grant No.201601D102004) and the Natural Science Foundation for Young Scientists of Shanxi Province, China (Grant No. 201601D021022), the Natural Science Foundation of Datong city (Grant No. 20150110).

Open Access This article is distributed under the terms of the Creative Commons Attribution 4.0 International License (http://creativecomm ons.org/licenses/by/4.0/), which permits unrestricted use, distribution, and reproduction in any medium, provided you give appropriate credit to the original author(s) and the source, provide a link to the Creative Commons license, and indicate if changes were made. Funded by $\mathrm{SCOAP}^{3}$.

\section{References}

1. A.G. Riess et al., Astron. J. 116, 1009 (1998)

2. S. Perlmutter et al., ApJ 517, 565 (1999)

3. A.G. Riess et al., Astron. J. 607, 665 (2004)

4. R.A. Knop et al., Astron. J. 598, 102 (2007)

5. A. Balbi et al., Astron. J. 545, L1 (2000)

6. A.H. Jaffe, PRL 86, 3475 (2001)

7. D.N. Spergel, ApJS 148, 175 (2003)

8. D.N. Spergel, ApJS 170, 377 (2007)

9. S. Weinberg, Rev. Mod. Phys. 61, 1 (1989)

10. B. Ratra, P.E.J. Peebles, PRD 37, 3406 (1988)

11. R. Caldwell, R. Dave, P.J. Steinhardt, PRL 80, 1582 (1998)

12. R.R. Caldwell, PLB 545, 23 (2002)

13. R.R. Caldwell, M. Kamionkowski, N.N. Weinberg, PRL 91, 071301 (2003)

14. B. Feng, X. Wang, X. Zhang, PLB 607, 35 (2005)

15. B. Feng, M. Li, Y. Piao, X. Zhang, PLB 634, 101 (2006)

16. Z.K. Guo, Y. Piao, X. Zhang, Y. Zhang, PLB 608, 177 (2005)

17. A.Y. Kamenshchik, U. Moschella, V. Pasquier, PLB 511, 265 (2001)

18. Z.-H. Zhu, A\&A 423, 421 (2004)

19. H. Zhang, Z.-H. Zhu, PRD 73, 043518 (2006)

20. A. Cohen, D. Kaplan, A. Nelson, PRL 82, 4971 (1999)

21. M. Li, PLB 603, 1 (2004)

22. A.R. Cooray, D. Huterer, ApJ 513, 95 (1999)

23. P. Astier, PLB (2000) (submitted). arXiv:astro-ph/0008306v1

24. J. Weller, A. Albrecht, PRL 86, 1939 (2001)

25. G. Efstathiou, MNRAS 342, 801 (2000)

26. M. Chavallier, D. Polarski, IJMPD 10, 213 (2001)

27. E.V. Linder, PRL 90, 091301 (2003)

28. Y. Wang, P.M. Garnavich, ApJ 552, 445 (2001)

29. C.R. Watson, R.J. Scherrer, PRD 68, 123524 (2003)

30. P.S. Corasaniti et al., PRD 70, 083006 (2004)

31. H.K. Jassal, J.S. Bagla, T. Padmanabhan, MNRAS 356, 11 (2005)

32. V.B. Johri, P.K. Rath, PRD 74, 123516 (2006)

33. E.M. Barboza, J.S. Alcaniz, Z.-H. Zhu, R. Silva, PRD 80, 043521 (2009)

34. K.I. Kellermann, Nature 361, 134 (1993) 
35. L.I. Gurvits, ApJ 425, 442 (1994)

36. J. Jackson, M. Dodgson, MNRAS 285, 806 (1997)

37. R. Vishwakarma, Class. Quantum Gravity 18, 1159 (2001)

38. Z.-H. Zhu, M.K. Fujimoto, ApJ 581, 1 (2002)

39. G. Chen, B. Ratra, ApJ 582, 586 (2003)

40. S. Cao, ApJ 806, 66 (2015a)

41. S. Cao, JCAP 02, 012 (2017a)

42. S. Cao, A\&A 606, A15 (2017b)

43. C. Blake, S. Brough, M. Colless, MNRAS 425, 405 (2012)

44. X. Xu, A.J. Cuesta, N. Padmanabhan, D.J. Eisenstein, C.K. McBride, MNRAS 431, 2834 (2013)

45. L. Samushia, B.A. Reid, M. White, MNRAS 439, 3504 (2014)

46. T. Delubac, J.E. Bautista, N.G. Busca, A\&A 574, A59 (2015)

47. E. De Filippis, M. Sereno, W. Bautz, G. Longo, ApJ 625, 108 (2005)

48. M. Biesiada, A. Piórkowska, B. Malec, MNRAS 406, 1055 (2010)

49. M. Biesiada, RAA 11, 641 (2011)

50. S. Cao, JCAP 03, 016 (2012)

51. S. Cao, G. Covone, Z.-H. Zhu, ApJ 755, 516 (2012)

52. S. Cao, Z.-H. Zhu, PRD 90, 083006 (2014)

53. S. Cao, ApJ 806, 185 (2015b)

54. S. Cao, N. Liang, Z.-H. Zhu, MNRAS 416, 1099 (2011)

55. S. Cao, Z.-H. Zhu, PRD 84, 023005 (2011)

56. S. Cao, N. Liang, IJMPD 22, 1350082 (2013)
57. S. Cao, Y. Chen, J. Zhang, Y.B. Ma, IJTP 54, 1492 (2015)

58. R.A. Preston, D.D. Morabito, J.G. Williams, J. Faulkner, D.L. Jauncey, G.D. Nicolson, Astrophys. J. 90, 1599 (1985)

59. X.L. Li et al., Testing and selecting cosmological models with ultracompact radio quasars. EPJC (submitted) (2017)

60. J.Z. Qi, EPJC 77, 502 (2017a)

61. P.A.R. Ade, A\&A 594, A14 (2016)

62. W. Hu, N. Sugiyama, ApJ 471, 542 (1996)

63. J.R. Bond, G. Efstathiou, M. Tegmark, MNRAS 291, L33 (1997)

64. A. Lewis, S. Bridle, PRD 66, 103511 (2002)

65. N. Suzuki, D. Rubin, C. Lidman, ApJ 746, 85 (2012)

66. A.G. Riess, ApJ 826, 56 (2016)

67. R. Jimenez, A. Loeb, ApJ 573, 37 (2002)

68. X.G. Zheng, X.H. Ding, M. Biesiada, ApJ 825, 17 (2016)

69. J. Z. Qi et al., What is parameterized $\operatorname{Om}(z)$ diagnostics telling us in light of recent observations? RAA (submitted) (2017)

70. J.V. Cunha, PRD 79, 047301 (2009)

71. V. Sahni, JETP Lett. 77, 201 (2003)

72. V. Sahni, A. Shafieloo, A.A. Starobinsky, PRD 78, 103502 (2008)

73. X.H. Ding, M. Biesiada, S. Cao, Z.X. Li, Z.H. Zhu, ApJL 803, L22 (2015)

74. R. Coppejans (2016). arXiv:1609.00575v1

75. S. Cao et al., Direct detection of cosmic acceleration by ultracompact high-redshift radio-quasars (in prep) (2017) 